BMJ

Open

Gastroenterology

\title{
Value and risk of percutaneous liver biopsy in patients with cirrhosis and clinical suspicion of autoimmune hepatitis
}

\author{
Pimsiri Sripongpun, ${ }^{1,2}$ Ananya Pongpaibul, ${ }^{3}$ Phunchai Charatcharoenwitthaya (i) ${ }^{1}$
}

Tocite:SripongpunP,PongpaibulA, Charatcharoenwitthaya P. Value and risk of percutaneous liver biopsy in patients with cirrhosis and clinical suspicion of autoimmune hepatitis. BMJ Open Gastro 2021;8:e000701. doi:10.1136/ bmjgast-2021-000701

Received 4 May 2021 Accepted 20 July 2021
Check for updates

\section{(C) Author(s) (or their} employer(s)) 2021. Re-use permitted under CC BY-NC. No commercial re-use. See rights and permissions. Published by BMJ.

${ }^{1}$ Medicine, Mahidol University Faculty of Medicine Siriraj Hospital, Bangkok, Thailand ${ }^{2}$ Medicine, Faculty of Medicine Prince of Songkla University, Hat Yai, Songkla, Thailand ${ }^{3}$ Pathology, Mahidol University Faculty of Medicine Siriraj Hospital, Bangkok, Thailand

Correspondence to Dr Phunchai Charatcharoenwitthaya; phunchai@yahoo.com

\section{ABSTRACT}

Objective The decision regarding whether to perform a liver biopsy in patients with cirrhosis and clinically suspected autoimmune hepatitis $(\mathrm{AlH})$ remains a challenge. This study aimed to assess the utility and complications of percutaneous liver biopsy in cirrhosis for differentiating AlH from other liver conditions.

Methods A clinicopathological database of patients undergoing percutaneous liver biopsies for suspected AlH (unexplained hepatitis with elevated $\gamma$-globulin and autoantibody seropositivity) was reviewed to identify patients presenting with cirrhosis. Biopsy slides were reviewed by an experienced hepatopathologist who was blinded to clinical data.

Results In 207 patients who underwent liver biopsy for suspected AlH, 59 patients (mean age: $59.0 \pm 12.0$ years, $83.1 \%$ female) had clinically diagnosis of cirrhosis. Mean Child-Turcotte-Pugh score was $6.6 \pm 1.6$, and $44 \%$ of patients had a Child-Turcotte-Pugh score $\geq 7$. According to the revised International AlH Group (IAIHG) criteria, histology assessment combined with clinical information facilitated a diagnosis of AlH or overlap syndrome of $\mathrm{AlH}$ and primary biliary cholangitis (PBC) in $81.4 \%$ of cases. Liver biopsy identified other aetiologies, including PBC $(n=2)$, non-alcoholic steatohepatitis $(n=6)$ and cryptogenic cirrhosis $(n=3)$. A reliable diagnosis of AlH could be made using histological category of the simplified criteria in $69.2 \%$ and $81.8 \%$ of cases using IAIHG scores before biopsy of $<10$ and $10-15$, respectively. Three patients with cirrhosis (5.1\%) experienced bleeding following biopsy, but none of 148 patients with non-cirrhosis had bleeding complication $(p=0.022)$.

Conclusion Liver biopsy provides important diagnostic information for the management of patients with cirrhosis and suspected $\mathrm{AlH}$, but the procedure is associated with significant risk.

\section{INTRODUCTION}

The International Autoimmune Hepatitis Group (IAIHG) affirmed the role of liver biopsy in the diagnosis of AIH in both their revised and simplified diagnostic criteria. ${ }^{1} 2$ Biochemical and immunological tests are insufficiently specific on their own to make a definite diagnosis of AIH. Given its wide range of clinical manifestations and characteristics, but not pathognomonic biochemical and immunological
Summary box

What is already known about this subject?

- The process of diagnosing autoimmune hepatitis (AlH) continues to be a challenge, in particular for those who present with manifestations of cirrhosis.

What are the new findings?

- Liver biopsy evaluation provides valuable histology information for discriminating AlH-related cirrhosis from other aetiologies; however, it carries a significant risk of bleeding following the procedure.

How might it impact on clinical practice in the foreseeable future?

- A liver biopsy provides additional essential diagnostic information that would enhance our understanding and management of unexplained advanced liver disease.

features, AIH can mimic other liver diseases. In patients with a chronic hepatitis pattern, some elderly women with non-alcoholic steatohepatitis (NASH)-related cirrhosis may show increased $\gamma$-globulin and positive autoantibodies. ${ }^{3} 4$ Thus-when using the diagnostic scoring systems for $\mathrm{AIH},{ }^{12}$ some patients with NASH may be misdiagnosed as having probable AIH. Hence, the process of diagnosing AIH continues to be a challenge, in particular for those who present with manifestations of cirrhosis.

A liver biopsy can identify patients with overlap syndromes, and is particularly useful in patients with biochemical cholestasis. A recent position statement by the IAIHG recommends that these patients should be classified and treated according to the predominant features of their disease. ${ }^{5}$ Moreover, liver biopsy provides prognostic information relating to the subsequent development of progressive fibrosis and the risk of liver-related death or transplantation, and it yields predictive information regarding response to treatment. Patients with AIHrelated cirrhosis or bridging necrosis on liver 
biopsy have a poorer prognosis than those without. ${ }^{6-9}$ However, these patients usually have steroid-responsive disease, so proactive treatment is warranted. Therefore, liver biopsy should be performed in all patients with clinical suspicion of AIH, and in patients with overlapping features between/among disorders within the spectrum of autoimmune liver diseases unless there is a significant contraindication for biopsy.

Numerous reports have described the safety and complication rate of percutaneous liver biopsy. Minor complications of the procedure include pain at the biopsy site and vasovagal episodes. ${ }^{10-16}$ The most common complication of major concern is bleeding, which occurs in $0.8 \%-1.7 \%$ of liver biopsies, ${ }^{10-18}$ and the reported mortality rate ranged from $0 \%$ to $0.14 \% .{ }^{10-16}$ The complication risk is presumed to be higher in patients with advanced liver disease, but detailed analyses of biopsy complications in patients with cirrhosis are scarce.

The main objective of this analysis was to assess the utility and safety of percutaneous liver biopsy for differentiating AIH from other conditions in patients with cirrhosis. A liver biopsy would provide additional essential diagnostic information that would enhance our understanding and management of unexplained advanced liver disease.

\section{METHODS}

\section{Study population}

We search clinical and liver biopsy databases to identify patients with a clinical suspicion of AIH in Siriraj Hospital (Bangkok, Thailand) during 2000-2015. The eligible criteria were as follows: (1) unexplained elevation of aminotransferase with elevated $\gamma$-globulin, and positive for the non-organ-specific antibodies known to be associated with AIH and (2) appropriate exclusion of other liver diseases, such as hepatitis B or C virus, alcohol, haemochromatosis, Wilson's disease and drug-induced hepatotoxicity. A total of 386 patients with suspected
AIH were identified. Empiric treatment with immunosuppressants was initiated for 144 patients who presented with acute liver failure $(n=22)$, acute severe hepatitis $(n=56)$, acute flares on chronic liver disease $(n=29)$, and cirrhosis with hepatic decompensation $(n=37)$, as shown in figure 1 . This group of patients with probable or definite AIH based on the 1999 revised IAIHG criteria were excluded due to the lack of histological evaluation before treatment. For the main objective of the study, 207 patients with available pathological slides for review were included in the analysis. Before the liver biopsy examination, the diagnosis of cirrhosis was established by the clinical manifestations, laboratory investigations and imaging studies.

\section{Clinical and laboratory evaluations}

Relevant clinical, biochemical and serological information at the time of liver biopsy were collected. Laboratory tests included serological markers, aspartate aminotransferase, alanine aminotransferase (ALT), alkaline phosphatase (ALP), total bilirubin, albumin, $\gamma$-globulin, immunoglobulins and standard haematological indices. Antinuclear antibody (ANA), smooth muscle antibody (SMA), liver-kidney-microsomal antibody and antimitochondrial antibody (AMA) were detected by indirect immunofluorescence, and a titre of 1:40 or greater was considered positive. The revised IAIHG score with and without histology assessment was calculated before the administration of specific therapy. ${ }^{1}$ Child-Turcotte-Pugh (CTP) score and the model for end-stage liver disease (MELD) score were calculated at the time of liver biopsy.

\section{Histological and clinicopathological diagnosis}

All biopsy slides were reviewed by a single experienced hepatopathologist (AP) who was blinded to all other clinical information. Histological features were categorised into one of the following three categories: atypical histology, histology compatible with AIH, or typical

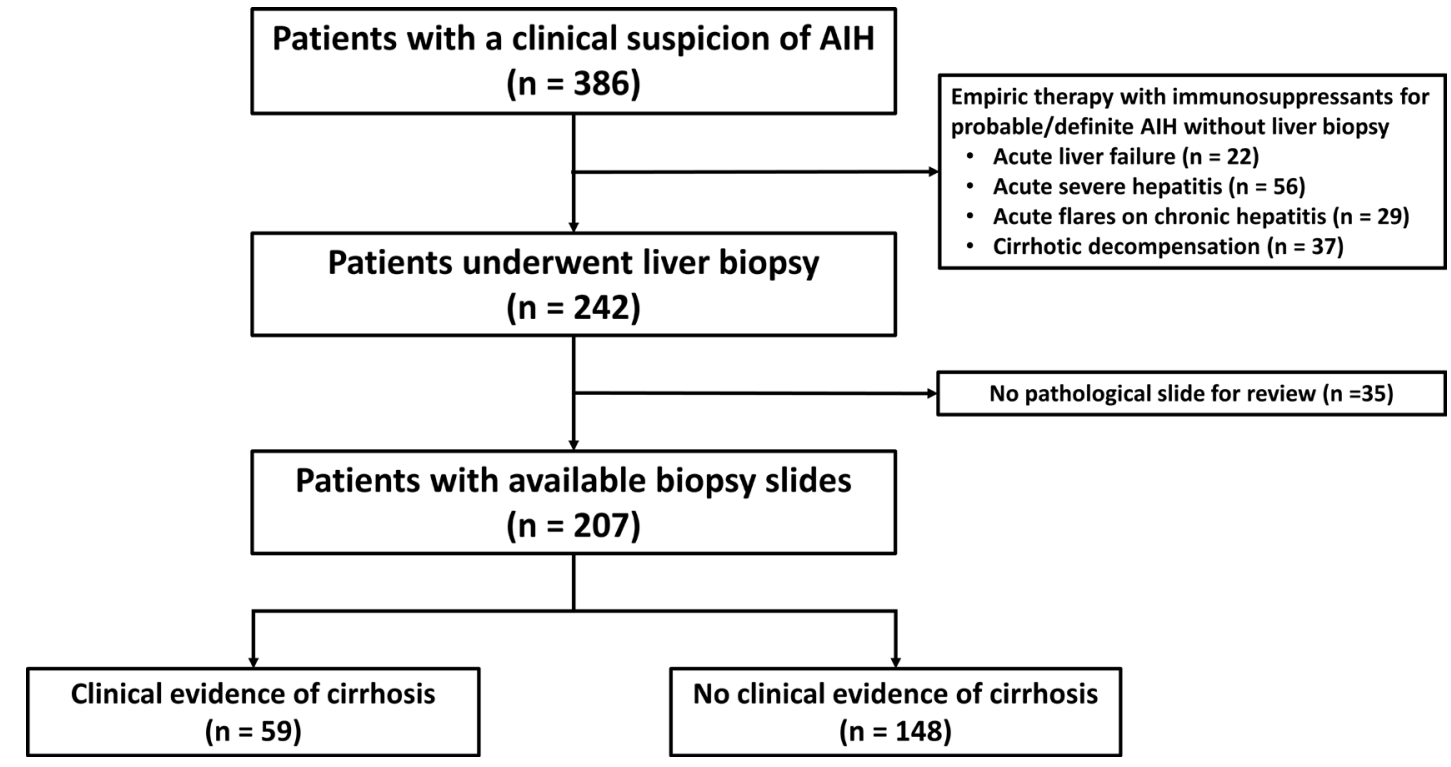

Figure 1 Flow chart of the study population. AlH, autoimmune hepatitis. 
histology according to the 2008 IAIHG simplified scoring system. ${ }^{2}$ In brief, the presence of interface hepatitis with lymphocytic or lymphoplasmacytic infiltrates in portal tracts and extending into the lobule, emperipolesis (active penetration by one cell into and through a larger cell), and hepatic rosette formation was considered to be typical AIH histology. Compatible feature was a finding of chronic hepatitis with lymphocytic infiltration without any of the features considered typical. Histology was considered atypical when there was evidence of an alternative diagnosis.

A diagnosis of AIH was established based on the 1999 revised IAIHG criteria. ${ }^{1}$ A diagnosis of primary biliary cholangitis (PBC) was made when at least two of the following three criteria were met: biochemical evidence of cholestasis based on ALP elevation; the presence of AMA; and/or histological evidence of non-suppurative destructive cholangitis and destruction of interlobular bile ducts. ${ }^{19}$ AIH-PBC overlap syndrome was diagnosed when two out of the following three PBC criteria (serum ALP level at least twice the upper limit of normal (ULN); presence of AMA; and/or liver histology showing florid bile duct lesion], and two out of the following three AIH criteria (serum ALT levels at least five times ULN; serum IgG levels at twice ULN or presence of SMA; and/or liver histology showing moderate or severe periportal or periseptal lymphocytic piecemeal necrosis) were met. ${ }^{20} \mathrm{~A}$ diagnosis of NASH was established by the presence of a characteristic pattern of steatosis, lobular inflammation, hepatocyte ballooning, Mallory-Denk body, and pericellular fibrosis at the centrilobular area on the liver biopsies in the absence of significant alcohol consumption. ${ }^{21}$

\section{Complications of liver biopsy}

To assess the risk of percutaneous liver biopsy among patients with cirrhosis, we compared the number and types of procedure-related complications in patients with suspected AIH-related cirrhosis with those of cases with suspected AIH but no clinical features of cirrhosis at biopsy evaluation during the same period. All adverse events that occurred within 30 days after liver biopsy were recorded and reviewed to determine their relationship with the biopsy. Serious adverse events were defined as complications requiring a blood transfusion, or complications that led to perforation of an organ, surgery, or death.

\section{Statistical analysis}

Categorical data are summarised as frequencies and percentages, and continuous data as mean (SD) or median (IQR) as appropriate. Statistical comparisons were performed using unpaired t-test or Mann-Whitney U-test for continuous variables, and Fisher's exact test or $\chi^{2}$ test for dichotomous variables. All statistical testing was performed at the conventional two-tailed $\alpha$ level of 0.05. The software package SPSS Statistics version V.18.0 (SPSS) was used for all analyses.

\section{RESULTS}

\section{Characteristics of the study cohort}

Of the 207 patients who underwent liver biopsy for suspected AIH, 59 patients had the clinical presentation of cirrhosis. The clinical characteristics of patients with cirrhosis are summarised in table 1 . The mean age at the time of the biopsy was $59.0 \pm 12.0$ years, and $49(83.1 \%)$ patients were female. The median level of serum ALT was $94 \mathrm{IU} / \mathrm{L}$ (IQR: 53-165), the mean level of $\gamma$-globulin was $4.8 \pm 1.1 \mathrm{~g} / \mathrm{dL}$. The mean MELD score was $10.8 \pm 4.5$. The average CTP score was $6.6 \pm 1.6$, and $56 \%$ and $44 \%$ of patients were in CTP class A and CTP class B, respectively. According to the 1999 revised IAIHG criteria, 46 patients (78\%) were classified as probable AIH (pre-biopsy IAIHG score of $10-15)$, and the remaining 13 patients (22\%) had a score less than 10 before liver biopsy evaluation (figure 2).

\section{Clinical and laboratory features}

By weighting of clinical, biochemical and histological parameters, 48 patients with cirrhosis satisfied the descriptive AIH criteria proposed by the IAIHG. Among those, 38 patients had a diagnosis of pure AIH, while ten patients were identified with AIH-PBC overlap syndrome. The remaining 11 patients had a diagnosis of PBC $(n=2)$, NASH $(n=6)$, or cryptogenic cirrhosis $(n=3)$. Clinical and laboratory features of AIH-related cirrhosis and other causes of cirrhosis at the time of biopsy are presented in table 1. There was no significant difference in the clinical characteristics of AIH patients versus those of patients with other liver diseases, as shown in table 1. Notably, ANA was often found in AIH patients than those with other liver diseases. In the subgroup of AIH cases, AIHPBC overlap syndrome patients were more likely to have a cholestatic pattern of liver enzymes with seropositivity for AMA, and less impaired hepatic function, as indicated by lower INR values and MELD scores than pure AIH patients. Patients with a pre-biopsy IAIHG score $<10$ were often diagnosed with AIH-PBC overlap syndrome compared with those with a pre-biopsy IAIHG score of $10-15(38.5 \%$ vs. $10.9 \%, \mathrm{p}=0.019)$.

\section{Comparisons of histological features between AlH and other liver diseases}

Significant overlap of histological findings was observed between AIH and other liver diseases (table 2). Interface hepatitis and lymphocytic/lymphoplasmacytic infiltrates in portal tracts with extension into the lobule were present in all cases of AIH and in almost patients with other liver diseases, but the level of severity was greater among AIH $(\mathrm{p}<0.05)$. Emperipolesis and rosette formation tended to be more frequent in AIH. AIH had more lobular inflammation, which is associated with a higher degree of injury $(\mathrm{p}=0.038)$. Steatosis $(\mathrm{p}=0.046)$ and Mallory-Denk body $(p=0.008)$ were features that significantly favoured NASH.

We also analysed 10 cases of AIH-PBC overlap syndrome vs 38 pure AIH to identify potentially discriminating 
Table 1 Baseline demographic and clinical characteristics of patients with cirrhosis compared between those with autoimmune hepatitis and those with other liver diseases

\begin{tabular}{|c|c|c|c|c|c|}
\hline \multirow[b]{2}{*}{ Characteristics } & \multicolumn{3}{|c|}{ Autoimmune hepatitis } & \multirow{2}{*}{$\begin{array}{l}\text { Other liver } \\
\text { diseases } \\
(n=11)\end{array}$} & \multirow[b]{2}{*}{ P value* } \\
\hline & $\begin{array}{l}\text { All AIH } \\
(n=48)\end{array}$ & $\begin{array}{l}\text { Pure AlH } \\
(\mathrm{n}=38)\end{array}$ & $\begin{array}{l}\text { AlH-PBC } \\
(n=10)\end{array}$ & & \\
\hline Age (years) & $58.6 \pm 12.3$ & $60.1 \pm 12.0$ & $53.0 \pm 12.5$ & $60.6 \pm 11.1$ & 0.625 \\
\hline Female gender & $39(81.3 \%)$ & $30(79.0 \%)$ & $9(90 \%)$ & $10(90.9 \%)$ & 0.670 \\
\hline Body mass index $\left(\mathrm{kg} / \mathrm{m}^{2}\right)$ & $23.8 \pm 3.8$ & $24.3 \pm 3.4$ & $22.5 \pm 4.8$ & $29.2 \pm 7.0$ & 0.089 \\
\hline \multicolumn{6}{|l|}{ Comorbidity } \\
\hline Diabetes mellitus & $6(12.5 \%)$ & $5(13.6 \%)$ & $1(10 \%)$ & $4(36.4 \%)$ & 0.079 \\
\hline Hypertension & $15(31.3 \%)$ & $12(31.6 \%)$ & $3(30 \%)$ & $5(45.5 \%)$ & 0.373 \\
\hline Dyslipidaemia & $6(12.5 \%)$ & $6(15.8 \%)$ & $0(0.0 \%)$ & $2(18.2 \%)$ & 0.635 \\
\hline Extrahepatic autoimmune disorder & $3(6.3 \%)$ & $3(7.9 \%)$ & $0(0.0 \%)$ & $1(9.1 \%)$ & 0.572 \\
\hline Total bilirubin (mg/dL) & $1.8(1.0-6.1)$ & $2.2(1.1-6.3)$ & $1.5(0.9-4.5)$ & $1.1(0.7-4.3)$ & 0.245 \\
\hline AST (IU/L) & $113(65-342)$ & $158(66-405)$ & $87(57-127) \dagger$ & $84(68-133)$ & 0.414 \\
\hline ALT (IU/L) & $102(50-228)$ & $110(52-290)$ & $88(48-141) \dagger$ & $72(55-137)$ & 0.454 \\
\hline ALP (IU/L) & $135(97-212)$ & 125 (96-192) & 230 (172-299)† & $117(92-186)$ & 0.763 \\
\hline Albumin (g/dL) & $3.4 \pm 0.7$ & $3.3 \pm 0.7$ & $3.5 \pm 0.6$ & $3.6 \pm 0.5$ & 0.274 \\
\hline Globulin (g/dL) & $4.8 \pm 1.2$ & $4.9 \pm 1.2$ & $4.7 \pm 1.1$ & $4.6 \pm 0.7$ & 0.586 \\
\hline Haemoglobin (g/dL) & $11.5 \pm 1.8$ & $11.6 \pm 1.8$ & $11.3 \pm 1.9$ & $11.9 \pm 1.6$ & 0.522 \\
\hline Platelet $\left(x 10^{9} / \mathrm{L}\right)$ & $189 \pm 65$ & $185 \pm 65$ & $189 \pm 86$ & $168 \pm 85$ & 0.405 \\
\hline INR & $1.13 \pm 0.16$ & $1.16 \pm 0.16$ & $0.99 \pm 0.09 \dagger$ & $1.06 \pm 0.20$ & 0.206 \\
\hline CTP score & $6.7 \pm 1.6$ & $6.9 \pm 1.6$ & $6.0 \pm 1.5$ & $6.1 \pm 1.3$ & 0.258 \\
\hline CTP class & & & & & 0.316 \\
\hline Class A & $25(52.1 \%)$ & $18(47.4 \%)$ & 7 (70\%) & $8(72.7 \%)$ & \\
\hline Class B & $23(47.9 \%)$ & $20(52.6 \%)$ & $3(30 \%)$ & $3(27.3 \%)$ & \\
\hline MELD score & $11.0 \pm 4.5$ & $11.5 \pm 4.5$ & $8.9 \pm 4.2 \dagger$ & $9.8 \pm 4.5$ & 0.466 \\
\hline ANA titre $\geq 1: 40$ & $40(83.3 \%)$ & $31(81.6 \%)$ & $9(90 \%)$ & $6(54.6 \%)$ & 0.039 \\
\hline SMA titre $\geq 1: 40$ & $21(43.8 \%)$ & $18(47.4 \%)$ & $3(30 \%)$ & $6(54.6 \%)$ & 0.520 \\
\hline AMA titre $\geq 1: 40$ & $7(14.6 \%)$ & $3(7.9 \%)$ & $4(40 \%) \dagger$ & $0(0.0 \%)$ & 0.328 \\
\hline Pre-biopsy IAlHG score & $11.4 \pm 2.2$ & $11.6 \pm 2.1$ & $10.8 \pm 2.3$ & $11.3 \pm 1.8$ & 0.838 \\
\hline
\end{tabular}

Data presented as mean $\pm \mathrm{SD}$, median and IQR, or number and percentage

A $p<0.05$ indicates statistical significance

${ }^{*}$ Comparison between patients with all $\mathrm{AlH}$ and those with other liver diseases.

†A $\mathrm{p}<0.05$ for comparison between patients with pure AlH and those with AlH-PBC.

$\mathrm{AlH}$, autoimmune hepatitis; AlH-PBC, autoimmune hepatitis overlap with primary biliary cholangitis; ALP, alkaline phosphatase; ALT, alanine aminotransferase; AMA, antimitochondrial antibody; ANA, antinuclear antibody; AST, aspartate aminotransferase; CTP, Child-Turcotte-Pugh; IAIHG, International Autoimmune Hepatitis Group; INR, international normalised ratio; MELD, model of end-stage liver disease; SMA, smooth muscle antibody.

histological features (table 2). This subgroup analysis showed severity of inflammation and the frequency of AIH-specific findings to be comparable between pure $\mathrm{AIH}$ and AIH-PBC overlap syndrome. However, the florid duct lesion was found in only AIH-PBC cases. Ductopenia and granuloma in the portal area were more frequently observed in AIH-PBC overlap syndrome $(\mathrm{p}<0.05)$.

\section{The concordance rate of clinicopathological versus} histological diagnosis

As shown in table 3, $54(91.5 \%)$ of the 59 patients with cirrhosis were concordantly diagnosed with wellestablished clinicopathological and histological diagnosis by the simplified criteria for AIH and other liver diseases: $44(74.6 \%)$ patients with pure AIH or AIH-PBC overlap syndrome, and $10(16.9 \%)$ cases with other liver diseases, including NASH $(n=6)$, PBC $(n=2)$ and cryptogenic cirrhosis $(n=2)$. Assessing liver histology of NASH patients showed characteristic features of steatosis, lobular inflammation, hepatocyte ballooning, and Mallory-Denk bodies with pericellular fibrosis at the centrilobular area without significant portal inflammation and interface hepatitis. In two cases with PBC, liver biopsy demonstrated ductopenia and portal inflammation with plasma cell infiltrates accompanied by mild interface hepatitis. Treatment with 


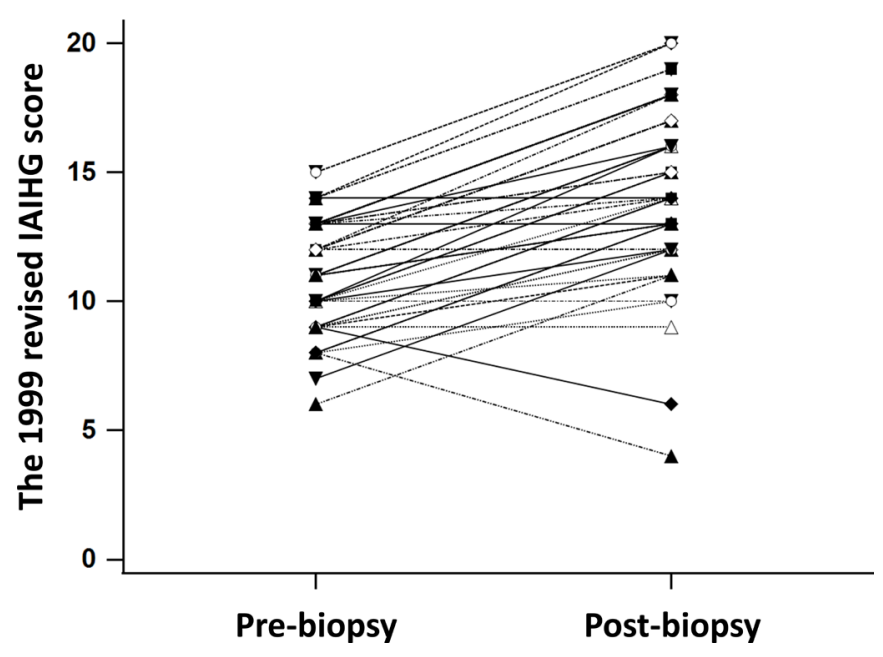

Figure 2 The revised 1999 International Autoimmune Hepatitis score before and after liver histological evaluation.

ursodeoxycholic acid led to biochemical improvement in both cases. Chronic hepatitis with mild lobular inflammation and cytological ballooning in the absence of significant steatosis was seen in two patients with diabetes, who were considered cryptogenic cirrhosis. According to the simplified criteria, these histological characteristics of patients with other liver diseases were considered atypical features for AIH.

Among discordant results, an older woman without comorbid disease was referred to evaluate abnormal liver tests, seropositivity for ANA, and cirrhotic features on abdominal ultrasonography. She was asymptomatic and did not use alcohol. A liver biopsy showed a mild degree of interface hepatitis and lymphocytic infiltrate in portal tracts, which were regarded as compatible features for AIH according to the simplified criteria. Fortunately, aminotransferase levels return to normal during follow-up despite no specific therapy. Hence, the exact cause of cirrhosis for this case is unknown. The other four patients with pure AIH or AIH-PBC overlap syndrome had prominent portal-based inflammation with interface hepatitis and characteristics of steatohepatitis, florid duct lesion and ductopenia, which were considered atypical AIH by the simplified criteria. However, the diagnosis of AIH and the overlap syndrome was made for these cases based on the combination of biochemical, immunological, and histological features and responsiveness to corticosteroid therapy or in combination with ursodeoxycholic acid.

Typical features and histology compatible with AIH were observed in $7.7 \%$ and $61.5 \%$ of patients with a prebiopsy IAIHG score $<10$, respectively; whereas, the same corresponding features were observed in $6.8 \%$ and $75 \%$ of those with a prebiopsy IAIHG score 10-15, respectively. No significant differences in histological features were observed between groups. AIH was finally diagnosed in $84.6 \%$ of patients in the pre-biopsy IAIHG score $<10$ subgroup, while $80.4 \%$ of cases with a prebiopsy IAIHG score of 10-15 were confirmed as having AIH.

\section{Liver biopsy complications}

Among 207 patients who underwent liver biopsy for suspected AIH, the cirrhotic group was older, and had lower levels of aminotransferase, albumin, haemoglobin and platelet but tended to have higher $\gamma$-globulin levels than the non-cirrhotic group (table 4). According to the 1999 revised IAIHG criteria, there was no significant difference in histological features between the cirrhotic and non-cirrhotic groups.

A liver biopsy complication was reported in 12 (5.8\%) of the 207 biopsies analysed (table 4 ). Three serious adverse events consisted of bleeding from the hepatic puncture site causing subcapsular haematoma with haemothorax in a case and haemoperitoneum in two patients. All of these patients had CTP class A cirrhosis. All patients required blood transfusion, and one patient underwent immediate intercostal drainage for haemothorax. Interestingly, the three patients with cirrhosis with bleeding complication had an INR of 1.1 or less with platelet counts greater than $120 \times 10^{9} / \mathrm{L}$. Although no serious adverse events were observed in patients with non-cirrhosis, six cases developed abdominal pain after the biopsy that required intravenous opioid analgesia in one case. No deaths occurred as a complication of liver biopsy.

\section{DISCUSSION}

In patients with cirrhosis and clinical suspicion of AIH, a standardised histological evaluation combined with clinical information is very useful for discriminating AIH from other chronic liver diseases. Nevertheless, liver biopsy is invasive and is potentially associated with severe complications that can even be life-threatening in some cases of advanced liver disease.

Liver biopsy provides valuable diagnostic information for the management of $\mathrm{AIH}^{22}$ Current international guidelines recommend performing a liver biopsy at the time of the first presentation in order to establish the diagnosis and to guide the treatment. ${ }^{23-26}$ Although the data in patients without cirrhosis showed that most patients with AIH features, based on clinical and laboratory characteristics, are likely to have compatible liver histology. Thus liver biopsy might not be necessary. ${ }^{27}$ Besides, treatment outcomes are comparable between patients with probable and definite AIH by pretreatment IAIHG score. ${ }^{28}$ However, the decision regarding whether to perform a percutaneous liver biopsy in patients with cirrhosis and clinical suspicion of AIH remains a challenge. In addition to having a potentially higher risk of procedure-related complications, patients with cirrhosis also have an increased risk of treatment-related adverse effects, which makes empirical treatment without a definite diagnosis perhaps equally risky. On the other hand, delayed diagnosis of AIH in patients with cirrhosis may lead to more rapid clinical deterioration than what would be observed in patients without cirrhosis. Hence, we set forth to explore the role and risk of liver biopsy evaluation in patients with cirrhosis and clinical suspicion of AIH. 
Table 2 Histological features of patients with cirrhosis compared between those with autoimmune hepatitis and those with other liver diseases

\begin{tabular}{|c|c|c|c|c|c|}
\hline \multirow[b]{2}{*}{ Histological features } & \multicolumn{3}{|c|}{ Autoimmune hepatitis } & \multirow{2}{*}{$\begin{array}{l}\text { Other liver } \\
\text { diseases } \\
(n=11)\end{array}$} & \multirow[b]{2}{*}{$P$ value* } \\
\hline & $\begin{array}{l}\text { All AlH } \\
(n=48)\end{array}$ & $\begin{array}{l}\text { Pure AlH } \\
(n=38)\end{array}$ & $\begin{array}{l}\text { AlH-PBC } \\
(n=10)\end{array}$ & & \\
\hline Interface hepatitis & & & & & $<0.001$ \\
\hline None & $0(0.0 \%)$ & $0(0.0 \%)$ & $0(0.0 \%)$ & $2(18.1 \%)$ & \\
\hline Mild & $9(18.8 \%)$ & $7(18.4 \%)$ & $2(20 \%)$ & $6(54.6 \%)$ & \\
\hline Moderate to severe & $39(81.2 \%)$ & $31(81.6 \%)$ & $8(80 \%)$ & $3(27.3 \%)$ & \\
\hline Emperipolesis & 7 (14.6\%) & $6(15.8 \%)$ & $1(10 \%)$ & $0(0.0 \%)$ & 0.329 \\
\hline Rosette formation & 7 (14.6\%) & $5(13.2 \%)$ & $2(20 \%)$ & $1(9.1 \%)$ & 0.634 \\
\hline Lymphocyte infiltration into the portal area & & & & & 0.007 \\
\hline None & $0(0.0 \%)$ & $0(0.0 \%)$ & $0(0.0 \%)$ & $1(9.1 \%)$ & \\
\hline Mild & $29(60.4 \%)$ & $23(60.5 \%)$ & $6(60 \%)$ & $10(90.9 \%)$ & \\
\hline Moderate to severe & $19(39.6 \%)$ & $15(39.5 \%)$ & $4(40 \%)$ & $0(0.0 \%)$ & \\
\hline Plasma cell infiltration into the portal area & & & & & $<0.001$ \\
\hline None & $2(4.2 \%)$ & $1(2.6 \%)$ & $1(10 \%)$ & $4(36.4 \%)$ & \\
\hline Mild & $25(52.1 \%)$ & $21(55.3 \%)$ & $4(40 \%)$ & $7(63.6 \%)$ & \\
\hline Moderate to severe & $21(43.7 \%)$ & $16(42.1 \%)$ & $5(50 \%)$ & $0(0.0 \%)$ & \\
\hline Lobular inflammation & & & & & 0.038 \\
\hline None & $7(14.6 \%)$ & $5(13.2 \%)$ & $2(20 \%)$ & $4(36.4 \%)$ & \\
\hline Few & $18(37.5 \%)$ & $14(36.8 \%)$ & $4(40 \%)$ & $5(45.5 \%)$ & \\
\hline Many & $23(47.9 \%)$ & $19(50 \%)$ & $4(40 \%)$ & $2(18.2 \%)$ & \\
\hline Steatosis & & & & & 0.046 \\
\hline$\leq 5 \%$ & 39 (81.2\%) & $30(78.9 \%)$ & $9(90 \%)$ & 5 (45.5\%) & \\
\hline$>5 \%-33 \%$ & $7(14.6 \%)$ & $6(15.8 \%)$ & $1(10 \%)$ & $5(45.5 \%)$ & \\
\hline$>33 \%-66 \%$ & $2(4.2 \%)$ & $2(5.3 \%)$ & $0(0.0 \%)$ & $1(9.1 \%)$ & \\
\hline Hepatocyte ballooning & & & & & 0.341 \\
\hline None & $23(47.9 \%)$ & $16(42.1 \%)$ & 7 (70\%) & 3 (27.3\%) & \\
\hline Few & $16(33.3 \%)$ & 15 (39.5\%) & $1(10 \%)$ & $4(36.4 \%)$ & \\
\hline Many & $9(18.8 \%)$ & 7 (18.4\%) & $2(20 \%)$ & $4(36.4 \%)$ & \\
\hline Glycogenated nuclei & $11(22.9 \%)$ & $11(28.9 \%)$ & $0(0.0 \%)$ & $5(45.5 \%)$ & 0.133 \\
\hline Mallory-Denk body & $14(29.2 \%)$ & $12(31.6 \%)$ & $2(20 \%)$ & 8 (72.7\%) & 0.008 \\
\hline Florid duct lesion & $3(6.3 \%)$ & $0(0.0 \%)$ & $3(30 \%)$ & $0(0.0 \%)$ & 1.000 \\
\hline Ductopenia & 7 (14.6\%) & $1(2.6 \%)$ & $6(60 \%) \dagger$ & $3(27.3 \%)$ & 0.376 \\
\hline Granuloma in portal area & $5(10.4 \%)$ & $0(0.0 \%)$ & $5(50 \%) \dagger$ & $0(0.0 \%)$ & 0.572 \\
\hline
\end{tabular}

Data presented as number and percentage

A $p<0.05$ indicates statistical significance

${ }^{*}$ Comparison between patients with all $\mathrm{AlH}$ and those with other liver diseases.

†A $\mathrm{p}<0.05$ for comparison between patients with pure $\mathrm{AlH}$ and those with AlH-PBC.

$\mathrm{AlH}$, autoimmune hepatitis; AlH-PBC, autoimmune hepatitis overlap with primary biliary cholangitis.

Our results revealed histological overlap between AIH and other chronic liver diseases. We observed that interface hepatitis and rosette formation, which are generally cited as classic histological features of AIH, could be observed in liver disease of variable aetiology. In 2008, the IAIHG condensed liver histology features into three categories as a necessary element for a diagnosis of AIH. We evaluated the ability of this histological system to discriminate between AIH and other chronic liver diseases. Given the observed concordance rate between histological and clinicopathological diagnosis (table 3), histological categories according to the simplified scoring system may be beneficial in the histological diagnosis of AIH.

Regarding comparison of AIH vs other liver diseases, lymphocytic infiltrates in portal tracts, another finding of chronic hepatitis pattern, was present in all patients with AIH and in almost patients with other hepatitides, but they were generally more severe in AIH. Additionally, 
Table 3 Agreement between clinicopathological diagnosis and histological diagnosis by the simplified criteria for autoimmune hepatitis and other liver diseases

Histological category by the simplified criteria*

\begin{tabular}{|lllll|} 
Clinicopathological diagnosis & $\mathbf{n}$ & Typical AlH (\%) & Compatible AlH (\%) & $\begin{array}{l}\text { Atypical AlH } \\
\text { (\%) }\end{array}$ \\
\hline AlH & 38 & $3(5.1)$ & $33(55.9)$ & $2(3.4)$ \\
\hline AlH-PBC overlap syndrome & 10 & $1(1.7)$ & $7(11.9)$ & $2(3.4)$ \\
\hline PBC & 2 & $0(0.0)$ & $0(0.0)$ & $2(3.4)$ \\
\hline NASH & 6 & $0(0.0)$ & $0(0.0)$ & $6(10.2)$ \\
\hline Cryptogenic & 3 & $0(0.0)$ & $1(1.7)$ & $2(3.4)$ \\
\hline
\end{tabular}

Data presented as number and percentage.

The agreement between clinicopathological diagnosis and histological diagnosis by the simplified criteria was shown in the grey boxes.

*The simplified scoring system for AlH condenses liver histology criteria into three categories: 'typical' when in addition to interface hepatitis, rosetting and emperipolesis are present, 'compatible' when not all three features are present, and 'atypical' when there is evidence of an alternative diagnosis.

$\mathrm{AlH}$, autoimmune hepatitis; $\mathrm{AlH}-\mathrm{PBC}$, autoimmune hepatitis overlap with primary biliary cholangitis; NASH, non-alcoholic steatohepatitis;

PBC, primary biliary cholangitis.

the prevalence of plasma cell-rich portal inflammation and lobular inflammation of variable severity were, in fact, higher in AIH than in other diseases. Ductopenia was detected in a small proportion of pure AIH and overlap syndrome, so their presence does not preclude a diagnosis of AIH. Glycogenated nuclei, Mallory-Denk body and steatosis or steatohepatitis are sometimes seen, but-if they are prominent—a diagnosis of AIH is less likely according to the revised IAIHG classification. ${ }^{1}$ Thus, even though the pattern of injury and typical

Table 4 Characteristics and complications compared between patients with cirrhosis and non-cirrhosis

\begin{tabular}{|c|c|c|c|}
\hline Variables & $\begin{array}{l}\text { Patients with } \\
\text { cirrhosis } \\
(n=59)\end{array}$ & $\begin{array}{l}\text { Patients with non- } \\
\text { cirrhosis } \\
(n=148)\end{array}$ & $P$ value \\
\hline \multicolumn{4}{|l|}{ Characteristics } \\
\hline Age (years) & $59.0 \pm 12.0$ & $54.5 \pm 13.2$ & 0.024 \\
\hline Female gender & $49(83.1 \%)$ & $134(90.5 \%)$ & 0.130 \\
\hline AST (IU/L) & 108 (67-279) & $262(95-621)$ & $<0.001$ \\
\hline ALT (IU/L) & $94(53-165)$ & $230(98-640)$ & $<0.001$ \\
\hline ALP (IU/L) & 135 (96-209) & 146 (96-200) & 0.949 \\
\hline Albumin (g/dL) & $3.4 \pm 0.7$ & $3.7 \pm 0.7$ & 0.004 \\
\hline Globulin (g/dL) & $4.8 \pm 1.1$ & $4.5 \pm 1.0$ & 0.059 \\
\hline Platelet $\left(\times 10^{9} / \mathrm{L}\right)$ & $186 \pm 68$ & $221 \pm 83$ & 0.005 \\
\hline Haemoglobin (g/dL) & $11.6 \pm 1.7$ & $12.3 \pm 1.5$ & 0.003 \\
\hline INR & $1.10(1.00-1.24)$ & $1.10(1.03-1.28)$ & 0.344 \\
\hline \multicolumn{4}{|l|}{ Liver histology } \\
\hline Interface hepatitis & 57 (96.6\%) & $140(94.6 \%)$ & 0.542 \\
\hline Predominantly lymphoplasmacytic infiltrate & $58(98.3 \%)$ & $142(95.9 \%)$ & 0.398 \\
\hline Rosette formation & $8(13.6 \%)$ & $20(13.5 \%)$ & 0.993 \\
\hline Biliary changes & $12(20.3 \%)$ & $22(14.9 \%)$ & 0.338 \\
\hline \multicolumn{4}{|l|}{ Complications of liver biopsy } \\
\hline All adverse events & $6(10.2 \%)$ & $6(4.1 \%)$ & 0.090 \\
\hline Pain at the hepatic puncture site & $4(6.8 \%)$ & $6(4.1 \%)$ & 0.410 \\
\hline Severe pain requiring intravenous analgesics & $2(3.4 \%)$ & $1(0.7 \%)$ & 0.141 \\
\hline Postbiopsy bleeding & $3(5.1 \%)$ & $0(0 \%)$ & 0.022 \\
\hline
\end{tabular}

Data presented as mean $\pm \mathrm{SD}$, median and IQR, or number and percentage

A $p<0.05$ indicates statistical significance

ALP, alkaline phosphatase; ALT, alanine aminotransferase; AST, aspartate aminotransferase; INR, international normalised ratio 
features of AIH are non-specific, the combined observation of inflammation (ie, types of infiltrates and area) and degree of inflammation can help discriminate AIH from other liver diseases.

Based on the descriptive criteria published by the IAIHG, ${ }^{1}$ none of our 59 patients with cirrhosis met the criteria for a definite diagnosis of AIH before histological assessment. Under this circumstance, it is reasonable to consider liver biopsy for differential diagnosis of AIH, since liver histology may show additional or unexpected findings that could affect patient management. This is supported by the fact that no clinical or laboratory features could be used to discriminate AIH-related cirrhosis from other aetiologies in our analysis. Overall, $48(81.4 \%)$ of our 59 patients had a final diagnosis of AIH after the detailed histology assessment. With information from liver histology, 6 (46.2\%) of 13 cases who initially had a prebiopsy IAIHG score $<10$ turned out to be AIH, and $32(69.6 \%)$ of 46 patients who were probable AIH by prebiopsy IAIHG scoring were definitely confirmed as AIH (figure 2). Furthermore, liver biopsy could identify 10 patients with features that overlap between disorders within the spectrum of autoimmune liver diseases, and biopsy was found to be particularly useful in those with biochemical cholestasis. Interestingly, biopsy-proven NASH was established in six cases who were misdiagnosed as having probable AIH by prebiopsy IAIHG scoring. The exact aetiology of cirrhosis cannot be identified in three patients after detailed clinical, laboratory and histological investigations. Some of these cases with seropositive autoantibodies may have an autoimmune nature. However, the benefit of immunosuppressive treatment in an asymptomatic older patient with the mild activity of interface hepatitis and lymphocyte infiltration in portal tracts on biopsy is not established; a decision not to treat was justified for such a case. In diabetic individuals with cirrhosis of unknown origin, the cause of cirrhosis might be a burnt out NASH; however, a significant lipid accumulation in the hepatocytes was not detected. Lifestyle change with improved physical activity and better nutrition was recommended for treating their metabolic conditions to optimistically slow or halt the progression of the liver disease. These findings highlight that histological evaluation is of enormous importance in managing patients with cirrhosis with clinical suspicion of AIH.

A decision whether or not to perform a liver biopsy must be made only after weighing the benefits against the risk of complications. In the present study, any adverse event occurred in $10.2 \%$ and $4.1 \%$ of patients in the cirrhotic and non-cirrhotic groups, respectively. Of the 12 adverse events observed in this study, only three serious bleeding events occurred in patients with compensated cirrhosis. The bleeding rate among patients with cirrhosis in our study was higher than the reported rates of $0 \%-0.6 \%$ in hepatitis $\mathrm{C}$ patients with advanced fibrosis/cirrhosis. ${ }^{15} 2930$ The disparity might be attributed to the fact that earlier studies that evaluated the risk of liver biopsy included patients with less advanced liver disease. Because the serious adverse events in our study involved bleeding, we evaluated for association with platelet count and INR value. We found that all bleeding complications were developed in patients with cirrhosis with normal platelet count and INR value. Hence, we recommend caution when considering liver biopsy in patients with cirrhosis, even in those with normal coagulation tests. Thromboelastography provides a more comprehensive global homeostasis assessment than conventional coagulation assays. ${ }^{31}$ There are limited data to suggest the clinical use of thromboelastography-guided correction of coagulation parameters before invasive procedures in patients with cirrhosis. ${ }^{32}$ Also, the utility of this point-of-care test has not been explored in those occurring procedurerelated bleeding. Further studies on the clinical application of viscoelastic instruments in cirrhosis are warranted.

This study has some limitations. First, the status of HLA-DR3 and HLA-DR4 was not assessed in our patients. Among 48 patients with $\mathrm{AIH}$, three patients had 15 points with histology assessment using the revised original pretreatment criteria in our group. The presence of HLA typing may upgrade some of them from probable AIH to definite diagnosis using the revised original criteria. Second, immunoglobulin G levels were not measured in most patients, so we could not calculate the diagnostic score for AIH regarding the simplified criteria. ${ }^{2}$

In conclusion, liver biopsy in patients with cirrhosis and suspected AIH provides valuable histological information on contributing to the clinical diagnostic scoring systems, identifying the overlap syndrome and differential diagnosis from other entities. However, it carries a significant risk of bleeding complications following the procedure. Thus, careful histological examination in conjunction with detailed clinical and laboratory information is essential for the optimal management of patients with cirrhosis and suspected AIH.

Contributors PC designed the study. PS, AP and PC participated in the data collection and analysis. PS and PC drafted this manuscript. PS, AP and PC interpreted the data. PC reviewed and revised this manuscript. All authors approved the final manuscript.

Funding This research was supported by a grant from the Siriraj Research Development Fund.

Competing interests None declared.

Patient consent for publication Not required.

Ethics approval The study protocol conformed to the ethical guidelines of the 1975 Declaration of Helsinki, and was approved by Siriraj Institutional Review Board (Si 082/2011). Given the retrospective nature of the research, the requirement for informed consent was waived.

Provenance and peer review Not commissioned; externally peer reviewed

Data availability statement Data are available on reasonable request. The data sets generated and/or analysed during the current study are available on reasonable request.

Open access This is an open access article distributed in accordance with the Creative Commons Attribution Non Commercial (CC BY-NC 4.0) license, which permits others to distribute, remix, adapt, build upon this work non-commercially, and license their derivative works on different terms, provided the original work is properly cited, appropriate credit is given, any changes made indicated, and the use is non-commercial. See: http://creativecommons.org/licenses/by-nc/4.0/. 
ORCID iD

Phunchai Charatcharoenwitthaya http://orcid.org/0000-0002-8334-0267

\section{REFERENCES}

1 Alvarez F, Berg PA, Bianchi FB, et al. International autoimmune hepatitis group report: review of criteria for diagnosis of autoimmune hepatitis. J Hepatol 1999;31:929-38.

2 Hennes EM, Zeniya M, Czaja AJ, et al. Simplified criteria for the diagnosis of autoimmune hepatitis. Hepatology 2008;48:169-76.

3 Adams LA, Lindor KD, Angulo P. The prevalence of autoantibodies and autoimmune hepatitis in patients with nonalcoholic fatty liver disease. Am J Gastroenterol 2004;99:1316-20.

4 Younes R, Govaere O, Petta S, et al. Presence of serum antinuclear antibodies does not impact long-term outcomes in nonalcoholic fatty liver disease. Am J Gastroenterol 2020;115:1289-92.

5 Boberg KM, Chapman RW, Hirschfield GM, et al. Overlap syndromes: the International autoimmune hepatitis group (IAIHG) position statement on a controversial issue. J Hepatol 2011;54:374-85

6 Al-Chalabi T, Underhill JA, Portmann BC, et al. Impact of gender on the long-term outcome and survival of patients with autoimmune hepatitis. J Hepatol 2008;48:140-7.

7 Feld JJ, Dinh H, Arenovich T, et al. Autoimmune hepatitis: effect of symptoms and cirrhosis on natural history and outcome. Hepatology 2005;42:53-62.

8 Cooksley WG, Bradbear RA, Robinson W, et al. The prognosis of chronic active hepatitis without cirrhosis in relation to bridging necrosis. Hepatology 1986;6:345-8.

9 Czaja AJ, Rakela J, Ludwig J. Features reflective of early prognosis in corticosteroid-treated severe autoimmune chronic active hepatitis. Gastroenterology 1988;95:448-53.

10 Cadranel JF, Rufat P, Degos F. Practices of liver biopsy in France: results of a prospective nationwide survey. for the group of epidemiology of the French association for the study of the liver (AFEF). Hepatology 2000;32:477-81.

11 Gilmore IT, Burroughs A, Murray-Lyon IM, et al. Indications, methods, and outcomes of percutaneous liver biopsy in England and Wales: an audit by the British Society of gastroenterology and the Royal College of physicians of London. Gut 1995;36:437-41.

12 Huang J-F, Hsieh M-Y, Dai C-Y, et al. The incidence and risks of live biopsy in non-cirrhotic patients: an evaluation of 3806 biopsies. Gut 2007;56:736-7.

13 Janes $\mathrm{CH}$, Lindor KD. Outcome of patients hospitalized for complications after outpatient liver biopsy. Ann Intern Med 1993;118:96-8.

14 Myers RP, Fong A, Shaheen AAM. Utilization rates, complications and costs of percutaneous liver biopsy: a population-based study including 4275 biopsies. Liver Int 2008;28:705-12.

15 Seeff LB, Everson GT, Morgan TR, et al. Complication rate of percutaneous liver biopsies among persons with advanced chronic liver disease in the HALT-C trial. Clin Gastroenterol Hepato 2010;8:877-83.

16 van der Poorten D, Kwok A, Lam T, et al. Twenty-Year audit of percutaneous liver biopsy in a major Australian teaching hospital. Intern Med J 2006;36:692-9.

17 Mahal AS, Knauer CM, Gregory PB. Bleeding after liver biopsy. West J Med 1981;134:11-14.

18 McGill DB, Rakela J, Zinsmeister AR, et al. A 21-year experience with major hemorrhage after percutaneous liver biopsy. Gastroenterology 1990;99:1396-400.

19 Lindor KD, Bowlus CL, Boyer J, et al. Primary biliary cholangitis: 2018 practice guidance from the American association for the study of liver diseases. Hepatology 2019;69:394-419.

20 Chazouillères $\mathrm{O}$, Wendum D, Serfaty L, et al. Primary biliary cirrhosis-autoimmune hepatitis overlap syndrome: clinical features and response to therapy. Hepatology 1998;28:296-301.

21 European Association for the Study of the Liver (EASL), European Association for the Study of Diabetes (EASD), European Association for the Study of Obesity (EASO). EASL-EASD-EASO clinical practice guidelines for the management of non-alcoholic fatty liver disease. J Hepatol 2016;64:1388-402.

22 Tiniakos DG, Brain JG, Bury YA. Role of histopathology in autoimmune hepatitis. Dig Dis 2015;33 Suppl 2:53-64

23 Manns MP, Czaja AJ, Gorham JD, et al. Diagnosis and management of autoimmune hepatitis. Hepatology 2010;51:2193-213.

24 Gleeson D, Heneghan MA, British Society of Gastroenterology. British Society of gastroenterology (BSG) guidelines for management of autoimmune hepatitis. Gut 2011;60:1611-29.

25 European Association for the Study of the L. EASL clinical practice guidelines: autoimmune hepatitis. J Hepatol 2015;63:971-1004.

26 Mack CL, Adams D, Assis DN, et al. Diagnosis and management of autoimmune hepatitis in adults and children: 2019 practice guidance and guidelines from the American association for the study of liver diseases. Hepatology 2020;72:671-722.

27 Björnsson E, Talwalkar J, Treeprasertsuk S, et al. Patients with typical laboratory features of autoimmune hepatitis rarely need a liver biopsy for diagnosis. Clin Gastroenterol Hepatol 2011;9:57-63.

28 Czaja AJ. Comparability of probable and definite autoimmune hepatitis by international diagnostic scoring criteria. Gastroenterology 2011;140:1472-80.

29 Sherman KE, Goodman ZD, Sullivan ST, et al. Liver biopsy in cirrhotic patients. Am J Gastroenterol 2007;102:789-93.

30 Pockros PJ, Jeffers L, Afdhal N, et al. Final results of a double-blind, placebo-controlled trial of the antifibrotic efficacy of interferongamma1b in chronic hepatitis $\mathrm{C}$ patients with advanced fibrosis or cirrhosis. Hepatology 2007;45:569-78.

31 O'Leary JG, Greenberg CS, Patton HM, et al. AGA clinical practice update: coagulation in cirrhosis. Gastroenterology 2019;157:34-43.

32 Shin KH, Kim IS, Lee HJ, et al. Thromboelastographic evaluation of coagulation in patients with liver disease. Ann Lab Med 2017;37:204-12. 